\title{
Beurteilung der Geschäftsfähigkeit und Testierfähigkeit bei Schlaganfallpatienten mit Aphasie
}

\section{Legal Capacity in patients with post-stroke-aphasia}

Autoren

Andrea Dreßing ${ }^{1}$, Cornelius Weiller ${ }^{1}$, Klaus Foerster ${ }^{2}$, Harald Dreßing ${ }^{3}$

Institute

1 Klinik für Neurologie und Neurophysiologie, Universitätsklinik Freiburg

2 Universitätsklinik für Psychiatrie und Psychotherapie, Tübingen

3 Zentralinstitut für Seelische Gesundheit, Medizinische Fakultät Mannheim, Universität Heidelberg

\section{Schlüsselwörter}

Aphasie, Innere Sprache, Schlaganfall, Geschäftsunfähigkeit, Begutachtung

Key words

aphasia, inner speech, stroke, legal capacity, assessment

eingereicht 04.07 .2018

akzeptiert 14.08.2018

Bibliografie

DOI https://doi.org/10.1055/a-0695-9104

Online-Publikation: 18.08.2018

Fortschr Neurol Psychiatr 2018; 86: 770-777

(c) Georg Thieme Verlag KG Stuttgart · New York

ISSN 0720-4299

Korrespondenzadresse

Dr. med. Andrea Dreßing M.A.

Klinik für Neurologie und Neurophysiologie

Universitätsklinik Freiburg

Breisacherstraße 64

79106 Freiburg

Tel.: 004976127050010

Fax: 0049761-270 53100

E-mail: andrea.dressing@uniklinik-freiburg.de

\section{ZUSAMMENFASSUNG}

Obwohl Aphasien nach ischämischen Schlaganfällen oder Hirnblutungen häufig auftreten, hat sich weder die medizinische Wissenschaft noch die obergerichtliche Rechtsprechung intensiver mit der praktisch bedeutsamen Frage beschäftigt, welche Auswirkungen diese Störung auf die Geschäftsfähigkeit hat.

Mit Hilfe moderner Bildgebungsstudien ist eine differenzierte Betrachtung der unterliegenden Pathologien der erworbenen Sprachstörungen, der daraus resultierenden unterschiedlichen klinischen Bilder der Aphasie sowie dem Erholungsverlauf möglich. Auf der Basis dieser Befunde sowie dem Konzept der „Inneren Sprache“ wird untersucht, inwieweit Aphasien die Geschäftsfähigkeit beeinträchtigen könnten und ein Phasenmodell zur Beurteilung der Geschäftsfähigkeit bei Aphasie vorgeschlagen, welches die grundsätzlichen Möglichkeiten der Erholung im Rahmen neuronaler Reorganisation berücksichtigt.

Die gutachtliche Beurteilung der Voraussetzungen zur Annahme einer Geschäftsfähigkeit oder -unfähigkeit bei Aphasie erfordert eine interdisziplinäre Zusammenarbeit von Neurologen, Psychiatern und Sprachtherapeuten. Da Gutachten zur Geschäftsunfähigkeit häufig ex-post erstellt werden müssen, ist der Gutachter auf eine detaillierte Befunddokumentation von Fähigkeiten und Defiziten bei Vorliegen einer Aphasie angewiesen. Um die bisherigen Limitationen in der Begutachtung der Geschäftsfähigkeit von Patienten mit Aphasien zu verbessern, wäre eine standardisierte Beschreibung der Aphasie und assoziierter neuropsychologischer Symptome als obligater Bestandteil eines Arztbriefes nach einer neurologischen Akut- und Rehabilitationsbehandlung wünschenswert.

\section{ABSTRACT}

Aphasia is a frequent cognitive disorder after stroke. Despite a high prevalence, there is comparatively little medical literature or high court jurisdiction concerning the question if aphasia impairs patients in their legal capacity.

Imaging studies allow a detailed understanding of the underlying pathology of aphasia, the resulting clinical pictures and potential mechanisms of recovery. Based on functional-anatomical models of language and associated concepts of inner speech, the impact of language disorders on legal capacity is evaluated. A phase model for the assessment of patients with aphasia is proposed, accounting for different stages of potential recovery after stroke. In practice, evaluation of the prerequisites for legal capacity or incapacity in patients with aphasia requires an interdisciplinary cooperation between neurologists, psychiatrists and speech language therapists. In cases of ex-post assessment, the evaluation of legal incapacity at a specific date, however, relies on a detailed documentation of the patient's language 
impairment and its recovery. To overcome the current limitations standardized and test-based assessment of neuropsychiatric and language capacities after acute hospital treatment and rehabilitation treatment is desirable.

\section{Einleitung}

Erworbene Sprachstörungen (Aphasien) treten häufig nach ischämischen Schlaganfällen oder Hirnblutungen auf, in Deutschland wird die Prävalenz der durch zerebrovaskuläre Ereignisse bedingten Aphasien auf ca. 70000 geschätzt, die Inzidenz neuer schwerer Aphasien nach Schlaganfällen auf rund 25000 pro Jahr [1]. Circa ein Drittel aller Schlaganfallpatienten leidet in der Akutphase unter einer Aphasie [2]. Aphasien beeinträchtigen die Patienten in ihrer alltäglichen Kommunikationsfähigkeit und Teilhabe. Trotz der hohen Prävalenz dieser Störungen hat sich weder die medizinische Wissenschaft noch die obergerichtliche Rechtsprechung intensiver mit der praktisch bedeutsamen Frage beschäftigt, welche Auswirkungen aphasische Störungen auf die Geschäftsfähigkeit haben [3]. Während zahlreiche Abhandlungen über die Beurteilung der Geschäftsfähigkeit im Rahmen psychiatrischer Erkrankungen vorliegen [4], werden die erworbenen Sprachstörungen eher kursorisch behandelt $[5,6]$. Offen bleibt, ob und in welchen Fällen eine erworbene Sprachstörung die juristischen Voraussetzungen der Geschäftsunfähigkeit erfüllt, die an den Begriff der freien Willensbildung geknüpft ist. In der vorliegenden Arbeit wird deshalb der Versuch unternommen, zu klären, welche neuropsychologischen und neuropsychiatrischen Symptome einer Aphasie gegebenenfalls die Voraussetzungen einer freien Willensbildung beeinträchtigen können. Mit Hilfe moderner neurobiologischer Forschungsmethoden ist eine differenzierte Betrachtung der unterliegenden Pathologien der erworbenen Sprachstörungen, der daraus resultierenden unterschiedlichen klinischen Bilder der Aphasie sowie dem Erholungsverlauf zum Teil möglich. Die Idee von Sprache als mögliche Form des Denkens („Innere Sprache“) wird dabei als konzeptueller möglicher Rahmen evaluiert. Auf der Basis dieser Befunde wird ein Phasenmodell zur Beurteilung der Geschäftsfähigkeit bei Aphasie vorgeschlagen.

\section{Rechtliche Grundlagen zur Geschäftsfähigkeit}

Geschäftsunfähig ist, wer sich in einem die freie Willensbestimmung ausschließenden Zustand krankhafter Störung der Geistestätigkeit befindet, sofern nicht der Zustand seiner Natur nach ein vorübergehender ist. ( $\S 104$, II BGB). Davon abzugrenzen ist die im §105 BGB geregelte Nichtigkeit der Willenserklärung. Dort heißt es: „... Nichtig ist auch eine Willenserklärung, die im Zustand der Bewusstlosigkeit oder vorübergehenden Störung der Geistestätigkeit abgegeben wird." Die Testierfähigkeit ist eine spezielle Ausprägung der Geschäftsfähigkeit auf dem Gebiet des Erbrechts, so dass die folgenden Ausführungen grundsätzlich sowohl für Begutachtung der Geschäfts- als auf für die Testierfähigkeit Anwendung finden können.

Unter den Begriff der freien Willensbestimmung lässt sich eine Vielzahl theoretischer Konzepte fassen; während die neurophilosophische Debatte um den freien Willen die mögliche
Determinierung des Willen durch neuronale Vorgänge ins Zentrum stellt, kann für die Beurteilung der Geschäftsfähigkeit der Begriff der freien Willensbestimmung auf die Frage reduziert werden, ob die Handlungs- und Entscheidungskompetenzen, die „normale Bestimmbarkeit einer Person durch vernünftige Erwägung“ (BHG, NJW 1937), beeinträchtigt sind.

Aus neuropsychiatrischer Sicht liegen die Voraussetzungen zur Annahme einer "freien Willensbestimmung" dann nicht vor, wenn eine Erkrankung oder Störung die Umsetzung persönlicher Wertvorstellungen verhindert, in dem sie entweder die kognitiven Voraussetzungen der Willensbildung, der Intentionsbildung und Prozesse der Intentionsinitiierung und -realisierung beeinträchtigt, oder indem sie die motivationalen Voraussetzungen der Willensbildung verändert, indem sie den Zugang zu Wertvorstellungen verstellt bzw. Wertgefüge oder affektive dynamische Grundlagen von Entscheidungsprozessen verformt. Dies kann beispielsweise bei qualitativen oder quantitativen Bewusstseinsstörungen, formalen oder inhaltlichen Denkstörungen, Psychosen, Demenzen oder schweren affektiven Erkrankungen der Fall sein $[7,8]$.

Habermeyer u. Saß formulieren zur freien Willensbildung jedoch weiter: „Eine zielgerichtete Entscheidung ist nur dann möglich, wenn der zugrunde liegende Sachverhalt bekannt ist bzw. wenn der Prozess der Entscheidungsbildung, das Für und Wider einer Entscheidung vergegenwärtigt werden kann." [7], und fokussieren damit auch auf die kognitiven und kommunikativen Aspekte der Willensbestimmung. Aphasien stellen in Bezug auf diesen Aspekt eine besondere Herausforderung dar. Im Folgenden sollen daher Aphasien hinsichtlich ihrer klinischen Manifestation, der zugrunde liegenden Neuroanatomie und dem zeitlichen Verlauf beleuchtet werden und es soll der Frage nachgegangen werden, ob und wann die kognitiven und/oder motivationalen Voraussetzungen zur freien Willensbildung durch erworbene Sprachstörungen beeinträchtigt sein können.

\section{Funktionell-anatomische Grundlagen und klinische Bilder der Aphasie}

Aphasien sind zentrale Sprachstörungen, die linguistisch als Beeinträchtigung in den verschiedenen Komponenten der Sprache zu beschreiben sind (Phonologie, Lexikon, Syntax, Semantik). Sie kennzeichnen sich durch einen Verlust der erworbenen Fähigkeiten des Sprachverständnisses und/oder der Sprachproduktion, es können also expressive (Produktion) und rezeptive Störungen (Verständnis) der Sprache unterschieden werden.

Aktuelle funktionell-anatomischer Modelle der neuronalen Sprachrepräsentation im Gehirn beschreiben eine komplexe neuronale Organisation in einem vornehmlich linkshemisphärisch lokalisierten Netzwerk aus frontalen, parietalen und temporalen Arealen, welche durch mehrere subkortikal gelegene lange 
Assoziationsfaserbahnen verbunden sind. Das Netzwerk gliedert sich dabei in einen dorsalen und einen ventralen Verarbeitungsweg (Zwei-Schleifen-Modell) [9-15]. Der dorsale Weg ist notwendig für die Sprachproduktion mit Umwandlung sensorischer Lautrepräsentationen in motorisch-artikulatorischen Output, inklusiver gelernter auch längerer Sequenzen, der ventrale Weg ermöglicht die Verarbeitung von sensorischen Lautrepräsentationen zu bedeutungsvollem Inhalt, damit das Sprachverständnis und ist notwendig für Kategorisierung und Abstraktion. Syntax erfordert das Zusammenspiel beider Wege („Sequenz und Struktur"). In der Kindesentwicklung kommt es zu einer Verlängerung des dorsalen Weges bis zum „Broca-Areal“, erst dadurch wird eine Interaktion zwischen beiden Wegen möglich, notwendig sowohl für die Entwicklung von schwierigerer Grammatik als auch für die Internalisierung der Sprache [15].

Die netzwerkbasierte Organisation von Sprache ermöglicht auch Erholung. In einer Langzeit-Verlaufsuntersuchung an Schlaganfallpatienten konnten unterschiedliche Phasen der Erholung innerhalb des ersten Jahres unterschieden werden: eine frühe Phase (0-4 Tage nach Infarkt), eine postakute Phase (ca. 2 Wochen nach Infarkt), und eine „Konsolidierungsphase” (4-12 Monate nach Infarkt) [16]. Der Reorganisationsprozess bei Aphasie nach ischämischem Schlaganfall schließt periläsonelle und kontraläsionelle Areals der beider Hemisphären ein [16-19].

\section{Theoretische Überlegungen zur freien Willensbestimmung bei Patienten mit Aphasie}

Eine kognitive Voraussetzung zur Willensbildung ist die Fähigkeit, einen Gedanken zu formulieren, ihn abzuwägen und mögliche Konsequenzen zu evaluieren. Ob solche Denkprozesse und Sprache unmittelbar miteinander verknüpft sind, ist eine bis dato ungelöste Kontroverse der modernen Neurowissenschaften. Das Verhältnis von Sprache und Denken wird in unterschiedlichen Zusammenhängen konzeptualisiert.

Ein hilfreiches Konstrukt, um das Verhältnis von Sprache und Denken auch auf neuronaler Netzwerkebene zu untersuchen ist das Konzept der „Inneren Sprache“. Unter Innerer Sprache (Inner Speech, Private Speech) versteht man die internalisierte lautlose Verwendung von Sprache [21, 22], die die Ressourcen des Sprachsystems im Sinne innerer Sprachrepräsentationen für komplexe kognitive Funktionen und Überlegungen nutzbar macht. Es wird dabei zwischen einer konkreten inneren Sprache (expanded inner speech) als Sprache ohne Artikulation und einer kondensierten abstrakten Form der inneren Sprache (condensed inner speech) mit reduzierter syntaktischer Komplexität [23] unterschieden.

Zahlreiche Ansätze und Untersuchungen schreiben der Inneren Sprache eine zentrale Rolle für die Kognition zu (siehe dazu $[24,25])$. Ein Ansatz ist, dass Innere Sprache die Inhalte des Kurzzeitgedächtnisses im Sinne innerer phonologischer Loops verfügbar hält [26, 27], andere Autoren konnten zeigen, dass Innere Sprache die exekutiven Funktionen und das Problemlösen unterstützt (z. B. Task-switching) [28]. Hier erscheinet v. a. Innere Sprache im Sinne von Sprechen ohne Laut relevant. Darüber hinaus ermöglicht Denken in linguistischen Kategorien im Sinne der abstrakten Innere Sprache vermutlich auch, unterschiedliche Inhalte des Bewusstseins zu sammeln, zu integrieren und komplexere oder konzeptuelle Gedanken zu formulieren $[29,30]$, Basis dafür könnte die Interaktion des dorsalen und ventralen Wegen des Sprachnetzwerkes sein [15].

Die Fähigkeit zur Inneren Sprache bei Patienten mit Aphasien ist bisher noch nicht umfassend erforscht. Einige Arbeiten konnten zeigen, dass Patienten mit Aphasien im Vergleich zu Gesunden auch eine Einschränkung der Inneren Sprache, insbesondere der konkreten (expanded) inneren Sprache aufweisen [31-33]. Die Einschränkungen der inneren und offenen Sprache dissoziieren jedoch teilweise auch, sodass von einer Sprachstörung nicht unmittelbar auf eine Störung der Inneren Sprache geschlossen werden kann. Die meisten der bisher vorliegenden Studien zeigen jedoch, dass es einen relevanten Zusammenhang zwischen innerer und offener Sprache gibt und die Innere Sprache wahrscheinlich auf einer intakten Prozessierung im dorsalen und ventralen Weg beruht. Patienten mit linkshemisphärischem Infarkt und einer Aphasie schnitten beispielsweise schlechter in Aufgaben ab, die die Fähigkeit zum Problemlösen forderten [34].

Bildgebungsstudien untermauern diese Hypothese. Der Vergleich zwischen Netzwerkaktivierungen bei innerer und offener Sprache findet weitgehend überlappende Aktivierungsmuster, es gibt jedoch auch dissoziierende Aktivierungen, auch wenn alle Studien mit der eingeschränkten Objektivierbarkeit und Messbarkeit der Innere Sprache konfrontiert sind und häufig Surrogatmarker der Innere Sprache verwenden [35-39]. Eine Läsionsstudie, die innere und offene Sprache bei Patienten nach linkshemisphärischen Schlaganfällen vergleicht, zeigt, dass Läsionen, die dem dorsalen Weg des klassischen Sprachnetzwerk zugeordnet werden (z. B. linker Gyrus frontalis inferior) auch zu Störungen der inneren Sprache (a.e. jedoch der konkreten Innere Sprache) führen können [40].

Ob Patienten mit schwerer Aphasie auch eine Einschränkung der kondensierten, abstrakten Inneren Sprache haben, verbleibt nach der aktuellen Studienlage ungeklärt. Basierend auf den anatomischen Kenntnissen kann jedoch die Hypothese aufgestellt werden, dass durch eine fehlende Interaktion zwischen dorsalen und ventralen Netzwerkanteilen die Fähigkeit reduziert ist, Sprache als Repräsentation abstrakter Konzepte und damit als Grundlage des Denkens nutzbar zu machen. Eine strukturelle Läsion des Sprachsystems und eine daraus resultierende höhergradige Einschränkung der Sprachfunktion, könnte so mittelbar - durch die Beeinträchtigung der Inneren Sprache - auch die grundlegenden Voraussetzungen der freien Willensbestimmungen beeinträchtigen, so dass es notwendig erscheint, sich mit möglichen Einschränkungen der Geschäftsfähigkeit bei Aphasikern im Einzelfall differenziert und ausführlich zu befassen.

Das Wissen um funktionell-anatomische Korrelationen ermöglicht es grundsätzlich, aus dem Schlaganfallmuster Rückschlüsse auf die möglichen Netzwerkbeeinträchtigungen und damit auf Symptome und Defizite zu ziehen [41], allerdings basieren die bisher durchgeführten Studien auf Gruppenanalysen und können daher bisher nicht mit ausreichender Genauigkeit auf den individuellen Fall angewendet werden. Für die Begutachtung von Schlaganfallpatienten bedeutet dies, dass allein aus den bildgebenden Befunden, die die Schlaganfalllokalisation aufzeigen, keine 
ausreichenden Aussagen über die daraus resultierenden Beeinträchtigungen möglich sind [42].

\section{Praktische Implikationen der freien Willensbestimmung bei Patienten mit Aphasie}

Neben den konzeptuellen Überlegungen des Zusammenhanges zwischen Sprachfähigkeit und Kognition zu den Voraussetzungen der Willensbildung ergeben sich bei Patienten mit Aphasien durch die Sprachstörung auch praktische Probleme: Patienten mit Aphasien sind eingeschränkt in ihrer Fähigkeit zu verstehen und/oder zu sprechen. Die kognitiven Voraussetzungen zur freien Willensbestimmung können dadurch beeinträchtigt werden. In einem ersten Schritt ist dabei - in Analogie zur Beurteilung der Einwilligungsfähigkeit zu Behandlungsmaßnahmen - die Beurteilung der folgenden Kriterien sinnvoll, auch wenn zu betonen ist, dass Einwilligungsfähigkeit und Geschäftsfähigkeit nicht miteinander vermischt werden dürfen, da Einwilligung eine auf eine konkrete Situation bezogene Handlung impliziert [43]. Folgende Kriterien können dabei erörtert werden: Der Patient muss einen bestimmten Sachverhalt verstehen können (Verständnis); er muss bestimmte Informationen, auch bezüglich der Folgen und Risiken, in angemessener Weise verarbeiten können (Verarbeitung); die Informationen müssen auch im Hinblick auf Alternativen, angemessen bewertet werden können (Bewertung) und zuletzt muss der eigene Willen auf der Grundlage von Verständnis, Verarbeitung und Bewertung der Situation bestimmt werden (Bestimmbarkeit des Willens).

Nimmt man zunächst diese Kriterien als Maßstab für die minimalen kognitiven Voraussetzungen der Willensbildung, ergeben sich zwei praktische Implikationen. Eine schwere Einschränkung des Sprachverständnisses, welche die kognitiven Voraussetzungen dahingehend einschränkt, dass externe Informationen nicht ausreichend verstanden werden können, um sie zur Willensbildung zu nutzen ist mit den neuropsychiatrischen Voraussetzungen zur Annahme einer freien Willensbestimmung nicht vereinbar. Der zweite Punkt von praktischer Relevanz ist die Formulierung des Willens bei Probanden mit schwerer Sprachproduktionsstörung. Der Wille, ist er gebildet, muss auch formuliert werden, sei es in sprachlicher oder in schriftlicher Form. Eine schwere expressive Sprachstörung, die Probanden daran hindert, dass sie ihren Willen ausreichend formulieren können, kann die Bestimmbarkeit des Willens in bedeutsamer Weise einschränken. Den Probanden verbleiben dann allenfalls kommunikative Fähigkeiten neben der Laut- und Schriftsprache wie etwa Mimik und Gestik. Letztere kann jedoch nach linkshemisphärischen Infarkten im Rahmen von Apraxien ebenfalls beeinträchtigt sein [44-46].

Um Geschäftsfähigkeit suffizient beurteilen zu können, ist der Gutachter demnach auf eine detaillierte Beschreibung der entsprechenden Sprachdefizite angewiesen. Die klinische Testung der Aphasie erfolgt im deutschsprachigen Raum mit Hilfe des Aachener Aphasie Tests (AAT) [20] bzw. des Aachener Aphasie Bedside Test (AABT) und des darin integrierten Token Tests, in dem die Unterkategorien Sprachverständnis, Benennen, Nachsprechen und Schriftsprache, sowie die Spontansprache mit
Kommunikationsverhalten, Artikulation \& Prosodie, Automatismen, Semantik, Phonematik, Syntax bewertet werden. Basierend auf diesen Tests können in der chronischen Phase unterschiedliche Aphasiesyndrome definiert werden (z. B. rezeptive Störungen mit Einschränkungen des Sprachverständnisses und expressive Störungen mit Einschränkungen der Sprachproduktion) der AABT dient der Testung in der akuten Phase. Der AAT sollte daher als Mindeststandart in der Testung während der chronischen Phase der Aphasie angewendet werden, da er eine altersadaptierte differenzierte Beurteilung der qualitativen Einschränkungen der Sprache und unterschiedlichen Teilbereichen sowie eine Beurteilung des Schweregrades (leicht, mittel, schwer) der Aphasie ermöglicht.

Weitere praktischen Implikationen berücksichtigt beispielsweise auch der Mental Capacity Act (2005) [47] im angelsächsischen Raum. Dieser fordert in solchen Konstellationen den Einsatz von vereinfachten Aufklärungsbögen mit Piktogrammen und eine standardisierte Prüfung der Fähigkeiten mit einem angepassten Bogen und unter Zuhilfenahme ausgebildeter Sprachtherapeuten [48-52]. Auf diese Weise könnte Einschränkungen im Sprachverständnis und in der Sprachproduktion auch bei der Beurteilung der Voraussetzungen der Geschäftsfähigkeit Rechnung getragen werden.

Der Einsatz standardisierter Tests, adaptierter Hilfsmittel und insbesondere die interdisziplinäre Beurteilung der Patienten durch Neurologen, Psychiater und Sprachtherapeuten könnte ein Mosaikstein in der Gesamtbeurteilung der Geschäftsfähigkeit von Aphasikern sein.

\section{Vorschlag eines Phasenmodells zur Beurteilung der Geschäftsfähigkeit bei Aphasien}

Entscheidend für die Beurteilung der Voraussetzungen der Geschäftsunfähigkeit bei Patienten mit Aphasie ist der Zeitpunkt der Beurteilung und das Ausmaß der durch die Aphasie bedingten neuropsychologischen und neuropsychiatrischen Funktionsstörungen. Eine kompetente Beurteilung setzt dabei eine enge interdisziplinäre Kooperation der Neurologie, der Logopädie und der Psychiatrie voraus. Eine ausführliche Befunddokumentation (s.o.) möglicher Funktionsstörungen in Hinblick auf eine Beeinträchtigung der Geschäftsfähigkeit ist dabei eine unabdingbare Voraussetzung. Daran mangelt es leider in der Praxis nicht selten, da in der Akutphase andere Aspekte verständlicherweise häufig Vorrang haben, aber auch in den Berichten der Rehakliniken die Thematik oft nicht ausreichend differenziert genug dargestellt wird. Grundsätzlich gilt jeder erwachsene Mensch als geschäftsfähig, die Voraussetzungen zur Annahme einer Geschäftsunfähigkeit müssen bewiesen werden. Dies ist nur auf dem Boden einer verlässlichen Dokumentation von Fähigkeiten und Defiziten möglich. Die oben skizzierten Studien legen es nahe, dass es bei Aphasien zu erheblichen Störungen neuronaler Netzwerke kommen kann, deren Funktionstüchtigkeit Grundlage der „Inneren Sprache“ ist, wobei deren Störung wiederum wesentliche Voraussetzungen einer freien Willensbildung beeinträchtigen kann. Sind die damit einhergehenden neuropsychologischen und neuropsychiatrischen Beeinträchtigungen und der 
Zeitpunkt, zu dem diese Beeinträchtigungen möglicherweise vorgelegen haben, nicht sorgfältig dokumentiert, kann es vorkommen, dass ein Aphasiker fälschlicherweise aus der in der gutachtlichen Praxis fast regelhaft vorkommenden ex-post Perspektive als geschäftsfähig eingeschätzt werden muss, wenn der Zeitpunkt des Rechtsgeschäfts in der Vergangenheit liegt oder die zu begutachtende Person bereits verstorben ist.

Einen zentralen Aspekt in der Beurteilung der Geschäftsfähigkeit von Aphasikern stellt also der Zeitpunkt der Beurteilung dar. Die netzwerkbasierte Organisation von Sprache (siehe oben) ermöglicht prinzipiell eine weitgehende Kompensation der Defizite bis hin zu einer restitutio ad integrum. Von einem chronischen und weitgehend stabilen Zustand kann in der Regel ab einem Zeitpunkt von ca. 12 Monaten nach dem Ereignis ausgegangen werden, zuvor unterliegt die Sprache einem dynamischen Erholungsprozess was bei der Begutachtung zu beachten ist. Die 12 Monatsfrist stellt dabei nur einen Anhaltspunkt dar, in der Praxis kann es Abweichungen sowohl nach oben als auch nach unten geben. Die Voraussetzungen zur Feststellung einer Geschäftsunfähigkeit sind in der Regel also frühestens 12 Monate nach dem Schlaganfallereignis gegeben, da im BGB für die Geschäftsunfähigkeit ein Zustand gefordert wird, der nicht nur vorübergehender Natur ist. Innerhalb der ersten 12 Monate nach einem Schlaganfall, der eine Aphasie zur Folge hat, kann also allenfalls die Frage bedeutsam werden, ob eine abgegebene Willenserklärung im Sinne des §105 BGB nichtig ist. Hierfür sind die oben dargestellten Kriterien (Verstehen/ Verarbeiten / Beurteilung / Willen bestimmen) als Beurteilungsleitfaden hilfreich.

In der Begutachtungspraxis der Geschäftsunfähigkeit ergeben sich meist die folgenden Konstellationen:

1. Die Frage der Geschäftsunfähigkeit wird innerhalb der ersten zwölf Monate nach dem Schlaganfall aufgeworfen, der Patient lebt noch und kann untersucht werden:

In dieser Konstellation ist darauf zu verweisen, dass die neuropsychiatrischen Voraussetzungen zur Annahme einer Geschäftsunfähigkeit in dieser Phase nicht festgestellt werden können, da aufgrund einer möglichen neuronalen Reorganisation festgestellte Funktionsbeeinträchtigungen unter Umständen nur vorübergehend sind. Es stellt sich in dieser Phase allenfalls die Frage einer möglichen Beeinträchtigung der Einwilligungsfähigkeit oder der Nichtigkeit einer Willenserklärung. Zur Beurteilung dieser Fragestellung sollten, unterstützt durch Sprachtherapeuten, die oben dargestellten Kriterien der Einwilligungsfähigkeit geprüft werden.

2. Die Frage der Geschäftsunfähigkeit wird mindestens 12 Monate nach dem Schlaganfall aufgeworfen, der Patient lebt noch und kann untersucht werden:

Bei dieser Konstellation ist zu unterscheiden, ob das Rechtsgeschäft in engem zeitlichen Kontext mit der aktuellen gutachtlichen Untersuchung steht (Konstellation 2a) oder ob das Rechtsgeschäft längere Zeit vor der aktuellen gutachtlichen Untersuchung getätigt wurde und ein aktuell erhobener Befund deshalb nicht ohne weiteres auf den Befund zum Zeitpunkt des Rechtsgeschäfts übertagen werden kann (Konstellation 2b). Konstellation 2a ist für die Begutachtung ideal. Mit Hilfe einer interdisziplinären Untersuchung, die insbesondere auch sprachtherapeutische Untersuchungstechniken einbeziehen muss, kann die Frage nach einer möglichen Geschäftsunfähigkeit in der Regel eindeutig beantwortet werden. Dabei ist auch der Verlauf zu berücksichtigen und der aktuelle Befund sollte zu den Befunden, die in den ersten 12 Monaten nach dem Schlaganfallereignis dokumentiert wurden in Beziehung gesetzt werden. Bei Konstellation 2b ist darauf hinzuweisen, dass der aktuell erhobene Befund möglicherweise nicht identisch ist, mit dem Befund, der zu dem Zeitpunkt des Rechtsgeschäfts vorlag. Da wesentliche Prozesse der Erholung und Neuorganisation neuronaler Netzwerke bei Aphasien aber innerhalb der erstens 12 Monate nach dem akuten Ereignis stattfinden, ist der aktuelle erhobene Befund durchaus wegweisend. Sofern sich dabei keine relevanten neuropsychologischen und neuropsychiatrischen Symptome nachweisen lassen, die die Voraussetzungen der Geschäftsfähigkeit beeinträchtigen, ist auch für den Zeitpunkt des möglicherweise länger zurückliegenden Rechtsgeschäfts-das aber mindestens 12 Monate nach dem Schlaganfallereignis getätigt wurde- von erhaltener Geschäftsfähigkeit auszugehen. Dies gilt auch für den umgekehrten Fall, wenn also zum Untersuchungszeitpunkt durch den Gutachter auch die Voraussetzungen der Geschäftsunfähigkeit aufgrund einer Aphasie festgestellt wurden. Dann ist auch von Geschäftsunfähigkeit zum früheren Zeitpunkt auszugehen, wenn es keine Anhaltspunkte dafür gibt, dass in der Zwischenzeit eine erneute zerebrale Schädigung eingetreten ist, sondern von einem chronischen Zustand auszugehen ist.

3. Die Frage der Geschäftsunfähigkeit wird für ein Rechtsgeschäft aufgeworfen, das mindestens 12 Monate nach dem Schlaganfall getätigt wurde, der Patient ist aber zwischenzeitlich verstorben und kann nicht untersucht werden: Hierbei handelt es sich um eine durchaus gängige und äußerst anspruchsvolle gutachtliche Fragestellung, die auf dem Boden vorhandener medizinischer Dokumentationen und von Zeugenaussagen vorgenommen werden muss. Grundsätzlich sind dabei folgende Konstellationen denkbar und zu beachten (3a und 3b). Bei Konstellation 3a ergeben die medizinischen Dokumente z. B. die Beschreibung einer schweren sensomotorischen Aphasie innerhalb der ersten Monate nach dem Schlaganfall. Mit Hilfe sprachtherapeutischer Testverfahren ist auch eine Aphasie-bedingte Störung der Einwilligungsfähigkeit dokumentiert und für bestimmte Aufgabenbereiche wurde deshalb auch ein gesetzlicher Betreuer eingesetzt. Der weitere Verlauf ist nicht gut dokumentiert. Eine differenzierte sprachtherapeutische Untersuchung hat 12 Monate nach dem Schlaganfall nicht mehr stattgefunden. Die Betreuung wurde im Verlauf ohne detailliert nachvollziehbare Begründung aufgehoben, weil der Patient in der Familie versorgt wurde. Die Zeugenaussagen sind bezüglich vorhandener Fähigkeiten und Beeinträchtigungen widersprüchlich. Bei dieser Konstellation 3a wird der Gutachter voraussichtlich aus folgenden Gründen zur Annahme einer Geschäftsunfähigkeit auch für den Zeitraum ab dem 12. Monat nach dem Schlaganfallereignis kommen: Innerhalb der ersten 12 Monate wurde eine gravierende Beeinträchtigung kognitiver Funktionen als Folge der Aphasie festgestellt, die so ausgeprägt war, dass von einer 
aufgehobenen Einwilligungsfähigkeit ausgegangen wurde. Sofern diese Beeinträchtigungen länger als 12 Monate angehalten hätten, wären auch die Voraussetzungen zur Annahme einer Geschäftsunfähigkeit erfüllt gewesen. Eine Besserung der Beeinträchtigungen ist zwar auch nach 12 Monaten grundsätzlich noch möglich, müsste aber in der vorliegenden Konstellation nachgewiesen werden. Wenn dies bei fehlender Dokumentation und widersprüchlichen Zeugenangaben auch bei Ausschöpfung aller möglichen Quellen nicht möglich ist, wird der Gutachter zur Annahme einer andauernden Geschäftsunfähigkeit kommen. Aus normativer Sicht stellt sich für das Gericht die Frage der Beweislastumkehr.

\section{KURZE KLINISCHE FALLVIGNETTE}

Ein Patient erleidet einen linkshemisphärischen Insult mit Hemiparese rechts und ausführlich dokumentierter sensomotorischer Aphasie. Im psychopathologischen Befund sind Desorientierung, Verwirrtheit und psychomotorische Unruhe dokumentiert. In einem Betreuungsgutachten wird u. a. festgehalten, dass eine Betreuungsnotwendigkeit für alle Bereiche vorliegt und der Patient in seiner freien Willensbildung beeinträchtigt ist. Der Patient wird später in der Familie des Sohns versorgt, ausführliche Befunddokumentationen liegen für die Zeit nach der Akut- und Rehabehandlung nicht mehr vor. Eine durchgreifende Befundbesserung nach 12 Monaten ist nirgendwo fachärztlich dokumentiert. 18 Monate nach dem Schlaganfall errichtet der Patient dennoch ein Testament, in dem er den Sohn zum Alleinerben bestimmt, obwohl weitere Kinder vorhanden sind. Sechs Monate nach Testamentserrichtung verstirbt der Patient. Es soll ein Gutachten zur Testierfähigkeit erstellt werden, da die Gültigkeit des Testaments von den Geschwistern angezweifelt wird. Dem Gutachter können nur ausführliche ärztliche Dokumentationen für eine Zeit bis etwa 12 Monate nach dem Schlaganfall vorgelegt werden, aus denen sich die Annahme einer Testierunfähigkeit ergibt. Die vom Sohn behauptete Besserung der Symptomatik zum Zeitpunkt der Testamentserrichtung ist nirgendwo ärztlich dokumentiert, und wird von einigen Zeugen aus dem Familienkreis bestritten, von anderen bestätigt. Die Aussagen der Familienmitglieder lassen sich auch bei deren Befragung durch den Gutachter in Anwesenheit des Gerichts nicht substantiieren. Aus gutachtlicher Sicht wird man feststellen müssen, dass die Voraussetzungen zur Annahme einer Testierunfähigkeit 12 Monate nach dem Schlaganfallereignis vorgelegen haben. Auch unter Ausschöpfung aller Quellen ist eine Besserung dieses Zustandes nicht nachzuweisen, so dass vom Vorliegen der Voraussetzungen zur Annahme einer Testierunfähigkeit zum Zeitpunkt der Testamentserrichtung auszugehen ist.

Bei Konstellation 3b ist in den medizinischen Unterlagen zwar eine sensomotorische Aphasie beschrieben. Die sich daraus ergebenden neuropsychologischen und neuropsychiatrischen Beeinträchtigungen sind aber nicht detailliert beschrieben, und es findet sich in den Unterlagen auch kein detaillierter sprachtherapeutischer Befund. Dasselbe gilt für den Entlassungsbericht aus der Rehaklinik. Zeugenaussagen sind in der Regel bei diesen Fragestellungen wenig hilfreich, da sie fast immer viel zu undifferenziert sind. Bei dieser Konstellation wird der Gutachter die Voraussetzungen einer Geschäftsunfähigkeit nicht beweisen können. Mögliche Zweifel, die vielleicht aufgrund gravierender struktureller Schädigungen des Gehirns in der Bildgebung aufkommen könnten, sind nicht geeignet, die Voraussetzungen der Geschäftsunfähigkeit festzustellen. Solange die Voraussetzungen der Geschäftsunfähigkeit nicht mit an Sicherheit grenzender Wahrscheinlichkeit vom Gutachter festgestellt werden können, ist nämlich von Geschäftsfähigkeit auszugehen. Konstellation 3b ergibt sich in der gutachtlichen Praxis häufig, was die Notwendigkeit einer differenzierten Befundbeschreibung funktioneller Auswirkungen einer Aphasie auf die Voraussetzungen der Einwilligungs- und Geschäftsfähigkeit unterstreicht.

\section{FAZIT FÜR DIE PRAXIS}

Die Ergebnisse funktionell-anatomischer Modelle der aktuellen neurowissenschaftlichen Sprachforschung und die Konzepte der inneren Sprache erlauben keinen automatischen Rückschluss im Hinblick auf die Geschäftsfähigkeit von Probanden mit Aphasie, geben aber Anlass dazu, im Falle einer schweren Aphasie die Voraussetzungen zur Geschäftsfähigkeit im Einzelfall differenziert zu prüfen. Da der Zeitpunkt des Schlaganfalls und des Rechtsgeschäfts dabei relevant ist, wird in dieser Arbeit ein Phasenmodell zu Prüfung der Geschäftsfähigkeit vorgeschlagen, bei der eine enge interdisziplinäre Zusammenarbeit zwischen Neurologen, Psychiatern und Sprachtherapeuten notwendig ist. Insbesondere in Fällen einer ex-post-Begutachtung ist eine sachgerechte Einschätzung dabei in den meisten Fällen nur auf dem Boden einer differenzierten Befunddokumentation möglich. Daher wäre es wünschenswert, wenn eine standardisierte Befundbeschreibung der Aphasie sowie neuropsychologischer und neuropsychiatrischer Symptome in Form eines detaillierten psychopathologischen Befund, einer logopädische Sprachbeurteilung, einer neuropsychologische Testung der darüber hinausgehenden Defizite (z. B. Praxie, Aufmerksamkeit) sowie ein neurologischer Status obligater Bestandteil eines Arztbriefes nach einer neurologischen Akut- und Rehabilitationsbehandlung würde. Patienten mit einer Aphasie kann der Rat erteilt werden, ein notarielles Testament mit sprachtherapeutischer und neurologisch-psychiatrischer Unterstützung und Begleitung zu errichten. In Anbetracht der demografischen Entwicklung werden sich gutachtliche Fragen zur Geschäftsfähigkeit bei Aphasien in Zukunft nämlich häufiger stellen.

Interessenkonflikt

Die Autoren (A.D., C.W., K.F., H.D.) geben an, dass keine Interessenkonflikte bestehen. 
Literatur

[1] Huber W, Poeck K, Springer L. Klinik und Rehabilitation der Aphasie: eine Einführung für Therapeuten, Angehörige und Betroffene. Stuttgart: Georg Thieme Verlag; 2006.

[2] Engelter ST, Gostynski M, Papa S et al. Epidemiology of Aphasia Attributable to First Ischemic Stroke: Incidence, Severity, Fluency, Etiology, and Thrombolysis. Stroke 2006; 37: 1379-1384.

[3] Cording C, Nedopil N. Psychiatrische Begutachtungen im Zivilrecht ein Handbuch für die Praxis. Lengerich: Pabst Science Publ; 2014.

[4] Dreßing H, Foerster K, Leygraf J et al. Begutachtung der Geschäfts- und Testierfähigkeit. Nervenarzt 2014; 85: 1441-1452.

[5] Widder B, Gaidzik PW, Bahemann A, Cording C, Dieterich M, Ernestus RI, Fabra M, Foerster K, Rauschelbach H-H, Jochheim K-A. Neurowissenschaftliche Begutachtung Gutachten in Neurologie und nicht-forensischer Psychiatrie. Stuttgart: Georg Thieme Verlag; 2018.

[6] Wetterling Tilmann. Freier Wille und neuropsychiatrische Erkrankungen Ein Leitfaden zur Begutachtung der Geschäfts- und Testierfähigkeit. 1. Auflage. Stuttgart: Kohlhammer; 2016.

[7] Habermeyer E, Saß H. Ein am Willensbegriff ausgerichteter, symptomorientierter Ansatz zur Prüfung der Geschäftsfähigkeit. Fortschritte der Neurol · Psychiatr 2002; 70: 5-10.

[8] Habermeyer E, Saß H. Die überdauernde krankhafte störung der geistestätigkeit als voraussetzung der geschäftsunfähigkeit. Nervenarzt 2002; 73: 1094-1099.

[9] Fridriksson J, Yourganov G, Bonilha L et al. Revealing the dual streams of speech processing. Proc Natl Acad Sci 2016; 113: 15108-15113.

[10] Fridriksson J, Den Ouden DB, Hillis AE et al. Anatomy of aphasia revisited. Brain 2018; 141: 848-862.

[11] Hickok G, Poeppel D. The cortical organization of speech processing. Nat Rev Neurosci 2007; 8: 393-402.

[12] Saur D, Kreher BW, Schnell S et al. Ventral and dorsal pathways for language. Proc Natl Acad Sci 2008; 105: 18035-18040.

[13] Catani M, Jones DK, Ffytche DH. Perisylvian language networks of the human brain. Ann Neurol 2005; 57: 8-16.

[14] Rauschecker JP. Ventral and dorsal streams in the evolution of speech and language. Front Evol Neurosci 2012; 4: 7.

[15] Rijntjes M, Weiller C, Bormann T et al. The dual loop model: its relation to language and other modalities. Front Evol Neurosci 2012; 4: 9.

[16] Saur D, Lange R, Baumgaertner A et al. Dynamics of language reorganization after stroke. Brain 2006; 129: 1371-1384.

[17] Crinion J, Price CJ. Right anterior superior temporal activation predicts auditory sentence comprehension following aphasic stroke. Brain 2005; 128: 2858-2871.

[18] Raja Beharelle A, Dick AS, Josse G et al. Left hemisphere regions are critical for language in the face of early left focal brain injury. Brain 2010; 133: $1707-1716$

[19] van Oers CAMM, Vink M, van Zandvoort MJE et al. Contribution of the left and right inferior frontal gyrus in recovery from aphasia. A functional MRI study in stroke patients with preserved hemodynamic responsiveness. Neuroimage 2010; 49: 885-893.

[20] Huber W, Poeck K, Willmes K, Weninger D. The Aachen Aphasia Test. 1984.

[21] Vygotsky LS. Thought and language, Revised And Expanded Edition. Cambridge (UK): MIT Press; 1962.

[22] Winsler A, Fernyhough C, Montero I. Private speech, executive functioning, and the development of verbal self-regulation. Cambridge: Cambridge University Press; 2009.

[23] Fernyhough C. Alien voices and inner dialogue: towards a developmental account of auditory verbal hallucinations. New Ideas Psychol 2004; 22: $49-68$
[24] Alderson-Day B, Fernyhough C. Inner speech: Development, cognitive functions, phenomenology, and neurobiology. Psychol Bull 2015; 141: 931-965.

[25] Perrone-Bertolotti M, Rapin L, Lachaux JP et al. What is that little voice inside my head? Inner speech phenomenology, its role in cognitive performance, and its relation to self-monitoring. Behav Brain Res 2014; 261: 220-239.

[26] Baddeley A. The episodic buffer: A new component of working memory? Trends Cogn Sci 2000; 4: 417-423.

[27] Baddeley A, Chincotta D, Adlam A. Working memory and the control of action: Evidence from task switching. J Exp Psychol Gen 2001; 130: 641-657.

[28] Emerson M], Miyake A. The role of inner speech in task switching: A dual task investigation. J Mem Lang 2003; 48: 148-168.

[29] Carruthers P. The cognitive functions of language. Behav Brain Sci 2002; 25(6): 657-74.

[30] Gauker C. Words and images: an essay on the origin of ideas. Oxford (UK): Oxford University Press; 2011.

[31] Geva S, Bennett S, Warburton EA et al. Discrepancy between inner and overt speech: Implications for post-stroke aphasia and normal language processing. Aphasiology 2011; 25: 323-343.

[32] Langland-Hassan P, Faries FR, Richardson M] et al. Inner speech deficits in people with aphasia. Front Psychol 2015; 6: 528.

[33] Stark BC, Geva S, Warburton EA. Inner Speech's Relationship with Overt Speech in Poststroke Aphasia. J Speech Lang Hear Res 2017; 60: 2406.

[34] Baldo J V., Dronkers NF, Wilkins D et al. Is problem solving dependent on language? Brain Lang 2005; 92: 240-250.

[35] Palmer ED, Rosen HJ, Ojemann JG et al. An event-related fMRI study of overt and covert word stem completion. Neuroimage 2001; 14: 182193.

[36] Huang J, Carr TH, Cao Y. Comparing cortical activations for silents and overt seech using event-related fMRI. Hum Brain Mapp 2001; 15: 39-53.

[37] Forn C, Ventura-Campos N, Belenguer A et al. A comparison of brain activation patterns during covert and overt paced auditory serial addition test tasks. Hum Brain Mapp 2008; 29: 644-650.

[38] Shuster LI, Lemieux SK. An fMRI investigation of covertly and overtly produced mono- and multisyllabic words. Brain Lang 2005; 93: 20-31.

[39] Basho S, Palmer ED, Rubio MA et al. Effects of generation mode in fMRI adaptations of semantic fluency: Paced production and overt speech. Neuropsychologia 2007; 45: 1697-1706.

[40] Geva S, Jones PS, Crinion JT et al. The neural correlates of inner speech defined by voxel-based lesion-symptom mapping. Brain 2011; 134: 3071-3082.

[41] Yourganov G, Smith KG, Fridriksson J et al. Predicting aphasia type from brain damage measured with structural MRI. Cortex 2015; 73: 203215.

[42] Dreßing A, Dreßing H. Possibilities and limitations of neurobiological research for forensic psychiatry and criminology. Monatsschrift fur Kriminologie und Strafrechtsreform 2014; 97.

[43] Kröber H-L. Psychiatrische Kriterien zur Beurteilung der Einwilligungsfähigkeit. Rechtsmedizin 1998; 8: 41-46.

[44] Vanbellingen T, Simonet R, Kübel $S$ et al. Impaired everyday gestural communication in apraxia: A reliable and valid short scale. Int J Stroke 2016; 11: 11-12.

[45] Hogrefe K, Ziegler W, Weidinger $\mathrm{N}$ et al. Non-verbal communication in severe aphasia: Influence of aphasia, apraxia, or semantic processing? Cortex 2012; 48: 952-962.

[46] Dressing A, Nitschke K, Kümmerer D et al. Distinct Contributions of Dorsal and Ventral Streams to Imitation of Tool-Use and Communicative Gestures. Cereb Cortex 2018; 28

[47] Department of Health (UK). Mental Capacity Act. 2005. 
[48] Ferguson A, Duffield G, Worrall L. Legal decision-making by people with aphasia: Critical incidents for speech pathologists. Int J Lang Commun Disord 2010; 45: 244-258.

[49] Aldous K, Tolmie R, Worrall L et al. Speech-language pathologists' contribution to the assessment of decision-making capacity in aphasia: A survey of common practices. Int J Speech Lang Pathol 2014; 16: 231-241.
[50] Stein J, Brady Wagner LC. Is Informed Consent a "Yes or No" Response? Enhancing the Shared Decision-Making Process for Persons with Aphasia. Top Stroke Rehabil 2006; 13: 42-46. 